\title{
Frequency Response of Second-Order Systems With Combined Coulomb and Viscous Damping ${ }^{1}$
}

\author{
Thomas A. Perls ${ }^{2}$ and Emile S. Sherrard
}

\begin{abstract}
Curves obtained with an analog computer are presented for the magnification factor versus frequency ratio of second-order systems with combined coulomb and viscous damping. The ranges of the parameters are as follows: Viscous damping ratio from 0.05 to 5.0 , in 15 steps; coulomb damping ratio from 0 to 0.9 , in 11 steps; and frequency ratio from 0 to 2.0 . Boundaries between regions with 0,1 , and 2 stops per half-cycle are also shown.
\end{abstract}

\section{Introduction}

The increasing importance of vibration measurements for both military and nonmilitary applications has stimulated the production of a large variety of vibration-measuring instruments in recent years. This activity in design and manufacture has not been matched by progress in the analysis of the response of vibration instruments, or in the extension or modernization of previous analyses of their response. It is still frequently assumed, in the application of these instruments, that the response of an actual vibration instrument is identical with the response of an ideal instrument in which the damping is entirely "viscous," i. e., proportional to the velocity of the mass or "seismic element." The actual response may differ significantly from the ideal response if the damping varies with other than the first power of the velocity, or is dependent on displacement, or if any appreciable amount of coulomb damping is present. This last type of damping, sometimes referred to as dry or sliding friction, exerts on the moving element a force that is constant in magnitude and always acts in the direction opposite to the velocity of the moving element.

Coulomb damping is usually present in a mechanical second-order system such as is illustrated in figure 1. Here, in addition to the coulomb damping, a spring force and a viscous damping force are shown as acting upon the seismic element. The motion of such a system has been analyzed by Den Hartog ${ }^{3}$ in a 1931 paper in which results are presented graphically in a series of figures. For application to vibration instruments in present use, Den Hartog's

1 This work was conducted under a program of basic instrumentation research and development sponsored by the National Bureau of Standards, the Office of Naval Research, the Air Research and Development Command and the Atomic Energy Commission.

2 Now at the Missile Systems Division ,Lockheed Aircraft Corp., Van Nuys, Calif.

J. P. Den Hartog, Forced vibrations with combined coulomb and viscou friction, Trans. Am. S oc. Mech. Eng. 53, APM 53-9, 107-115 (1931) results require extension to larger values of viscous friction and a larger range of frequencies.

The present paper extends these results through the ranges applicable to such inertial instruments as accelerometers and jerkmeters. These inertial instruments are usually designed for viscous damping between 0.6 and 0.7 of critical damping, and are usually employed between zero frequency and a maximum frequency less than the resonance frequency (of the mass and spring). Particular emphasis is therefore placed in this paper upon results for viscous damping between 0.6 and 0.7 of critical damping, and for frequencies between zero and the resonance frequency.

Results are presented graphically in figures 2 to 17. These curves extend from zero to twice resonance frequency, and are presented so as to be readily applicable to vibration instruments used below their resonance frequency. They were determined without particular difficulty through the use of an analog computer. The original program called for the computation of an additional set of curves for frequencies between twice the resonance frequency and 50 times the resonance frequency. These curves would have been applicable to devices such as seismometers and velocity meters, which are ordinarily employed at frequencies above their resonance frequency. Unfortunately, the analog. computer accuracy proved poor at these high frequencies. The computation of displacement at high frequencies consisted essentially of the double integration of the difference of a sinusoidal forcing function and the coulomb friction. When the analog. computer attempted this double integration, its results contained appreciable errors caused by drifting and hunting. The size of these errors caused a termination of the high-frequency computations. Hence, only curves applicable to vibration instruments usually employed below their resonance frequency are presented in this paper. 


\section{Differential Equation for Vibration Instruments}

Figure 1 shows a typical vibration instrument with its frame rigidly attached to a sinusoidally vibrating structure having a motion $X \cos \omega t$. The displacement, $x$, of the seismic element relative to the frame of the instrument satisfies the differential equation:

$$
M \ddot{x}+C \dot{x}+K x \pm F=M X \omega^{2} \cos \omega t,
$$

where (see fig. 1)

$M=$ mass of seismic element.

$C=$ viscous damping force per unit velocity.

$K=$ restoring force exerted by the spring per unit displacement.

$F=$ coulomb damping force, assumed constant in magnitude and changing sign so as always to oppose the motion.

$X=$ amplitude of forcing motion.

$\omega=2 \pi f=$ angular frequency of forcing motion.

A number of equations in Den Hartog's paper give a complete literal solution of this equation with the right-hand side of the equation replaced by a term $P \cos (\omega t+\varphi)$, where $P$ is the amplitude of a "periodic disturbing force." For practical application Den Hartog expressed his results by a set of computed curves of magnification factor versus frequency ratio for seven values of viscous damping ratio between 0 and 0.5 of critical damping, and for a number of values between 0 and 0.9 of the ratio of coulomb damping force to peak disturbing force. Most of Den Hartog's curves extend over a frequency range of 0.5 to 2.0 ; the one for zero viscous damping extends over a frequency range of 0 to 2.0. Curves for zero coulomb damping (i. e., viscous damping only) are also available elsewhere. ${ }^{4}$ In the present paper, all the curves extend over the frequency ratio range between 0 and 2.0. They are given for 15 values of viscous damping ratio between 0.05 and 5.0, and for 11 values of coulomb damping ratio between 0 and 0.9 .

\section{Summary of Results}

The curves shown in figures 2 to 17 are drawn through points plotted by an analog computer. Each figure presents a family of curves for a constant value of $C / C_{c}$, the ratio of viscous damping $C$, to critical viscous damping, $C_{c}=2 \sqrt{K M}$. Each curve of the family is a plot of magnification factor or acceleration response $x_{0} \omega_{n}^{2} / \ddot{X}$ versus the frequency ratio, $\omega / \omega_{n}$, where $x_{0}$ is the maximum value of the displacement $x$, $\omega_{n}=\sqrt{K / M}$ is the undamped resonance frequency of

«See, for example, C. S. Draper, W. McKay, and S. Lees, Instrument engineering. II. Mathematics (McGraw-Hili Book Co., Inc., New York, N. Y., 1953). the mass-spring system, and $\ddot{X}=\omega^{2} x$ is the peak acceleration of the forcing motion. For inertial instruments, the term "acceleration response" seems appropriate for $x_{0} \omega_{n}{ }^{2} / \ddot{X}$ because this quantity may be interpreted as the product of two important instrument characteristics: $x_{0} / \ddot{X}$, the response (displacement) of the seismic element per unit acceleration applied to the instrument, and $\omega_{n}^{2}$, the resonance frequency of the instrument, to which instrument sensitivity is inversely proportional.

Each curve of the family is drawn for a particular value of the parameter $a_{F} / \ddot{X}$, where $a_{F}$ is the minimum acceleration that must be applied to the instrument to overcome coulomb friction and produce motion. This parameter $a_{F} / \ddot{X}$ is equivalent to the parameter $F / P$ used by Den Hartog. Either may be obtained from the other by employing the relations $F=M a_{F}$ and $P=M \ddot{X}$.

The dashed line plotted in each figure is a boundary line between steady-state motions with no stop and one stop per half-cycle. In the region above this boundary, the steady-state motion is continuous without stops. In the region immediately below this boundary, the motion stops once each half-cycle.

Only these two regions, nonstop and one-stop motion, were computed for $C / C_{c} \geq 0.2$ and $\omega / \omega_{n} \geq 0.1$. For $C / C_{c} \leq 0.1$, four regions characterized by no stop, one stop, two stops, or more than two stops each half-cycle were computed. When more than two regions occur, the magnification factor versus frequency ratio curves become very irregular for $\omega / \omega_{n}$ less than about 0.4 . For $\omega / \omega_{n} \leq 0.1$, larger and larger amounts of computing time would be required for accurately determining the magnification factor as $C / C_{c}$ becomes smaller and smaller. Hence for $C / C_{c}=0.2,0.1$, and 0.05 , the curves are terminated at $\omega / \omega_{n}=0.1$.

\section{Accuracy of Results}

The curves of figures 2 to 17 are plotted to permit reading the magnification factor to within 1 percent of the full scale of each figure. These curves were drawn through points plotted by an analog computer. Over-all accuracy of the points plotted by the analog computer, which consisted of elements with an accuracy of \pm 0.1 percent of rated output, is estimated at \pm 0.3 percent of the full-scale magnification factor. A number of check points computed manually and by the computer agreed with each other to within \pm 1.0 percent. Total computer errors and drafting errors are believed to introduce, in any figure, inaccuracies of no more than 1 percent of the full-scale magnification factor of each figure.

It is believed that greater accuracy in computation would have resulted from the use of a digital computer that employed Den Hartog's literal equations. However, an estimate of total costs showed 
analog computation to be more economical. One reason was the availability in the analog computer's auxiliary equipment of a plotting board, which could partially process the results of the computer study by plotting points of the magnification-factor curves. The other important important advantage of the analog computer was its use of eq (1) rather than the involved literal equations of Den Hartog's paper, which would have been employed by the digital computer. Use of the analog computer avoided the expenditures necessary for a careful scrutiny of these equations for possible misprints.

\section{General Effects of Coulomb Friction Upon Instrument Response}

The presence of coulomb friction makes instrument response dependent on the amplitude of the sinusoidal excitation. This amplitude dependence is shown graphically in figure 18, which is a cross-plot of the data for $C / C_{c}=0.65$, as given in figure 10 . In figure 18, magnification factor is plotted versus $\ddot{X} / a_{F}$ for various constant values of $\omega / \omega_{n}$.

The asymptotes shown as horizontal lines in the right-hand portion of figure 18 represent the magnification factors at vibration amplitudes sufficiently large to make negligible the effect of coulomb friction. An inspection of the figure shows that, for $C / C_{c}=0.65$, $\ddot{X} / a_{F}$ must be $\geq 25$ if the actual response is to be within 5 percent of the asymptote for all values of $\omega \leq \omega_{n}$.

Rules similar to this can be stated for other values of $C / C_{c}$. For $C / C_{c} \geq 0.3$ and for $0 \leq \omega / \omega_{n} \leq 1.0$, a general rule of thumb for estimating the error introduced by coulomb friction is to assume that this error is equal to the error at $\omega / \omega_{n}=0$. According to this general rule, a given percentage value of $a_{F} / \ddot{X}$ results in that same percentage reduction of magnification factor from its ideal $\left(a_{F} / \ddot{X}=0\right)$ value. This rule is a good approximation for the reduction in response for $C / C_{c}$ in the range 0.6 to 0.7 . As the value of $C / C_{c}$ moves further and further outside this range, the approximation becomes poorer and poorer. However, the damping in most inertial instruments is between 0.6 and 0.7 of critical damping; hence, this rule is useful for practical inertial instruments.

A physical example of the effect of coulomb fraction upon instrument response is shown in figure 19 . This figure reproduces an oscillogram from two different velocity meters mounted back to back and subjected to the same sinusoidal forcing function. The upper trace shows that one instrument is stopping once each half-cycle as the result of coulomb friction. The lower trace shows the other to be exhibiting essentially ideal behavior.

Figure 20 shows the time variation of displacement and velocity for various values of $a_{F} / \ddot{X}$, for a low value of viscous friction $\left(C / C_{c}=0.1\right)$, and a low value of frequency ratio $\left(\omega / \omega_{n}=0.1\right)$. These records were made during the computer study. Each set of two traces is for a different value of $a_{F} / \ddot{X} . \quad$ At $a_{F} / \ddot{X}=0.3$, one-stop motion occurs, as evidenced by the long dwell of the velocity trace on the (central) zero line. For $a_{F} / \ddot{X}$ equal to 0.5 , the second trough of the damped sinusoidal oscillation in the velocity trace approaches the zero line. At $a_{F} / \ddot{X}=0.525$, this trough reaches the zero, and the motion stops twice during each half-cycle. Further increase of $a_{F} / \ddot{X}$ to 0.55 furnishes an additional restraint, which prevents another start after the motion stops at nearly the same point of the velocity trough. Thus we have again one stop per half-cycle. The motion remains one-stop until displacement and velocity decrease sufficiently for a stop to occur at the first trough of the velocity, giving two-stop motion once more. This occurs at approximately $a_{F} / \ddot{X}=0.8$. However, an increase of $a_{F} / \ddot{X}$ to 0.9 furnishes the additional restraint necessary to prevent another start. Thus motion again becomes one-stop.

As $C / C_{c}$ decreases from 0.1 , the damped sinusoid of velocity becomes less damped and it is conceivable that for $\omega / \omega_{n} \leq 0.1$, motion may stop not only at the third, second, or first trough of the velocity, but also at a fourth, fifth, or higher-order trough of the velocity. In such cases, the response of the instrument becomes very irregular.

The analog computer study was performed by W. McCool and B. Zimmerman of the Naval Research Laboratory. The authors thank Messrs. McCool and Zimmerman for their suggestions and excellent cooperation in this study. M. Abramowitz and R. Dressler supplied information on the use of the digital computer. W. A. Wildhack continually furnished stimulating ideas during the investigation. A. Bucek prepared the curves and illustrations shown in this report.

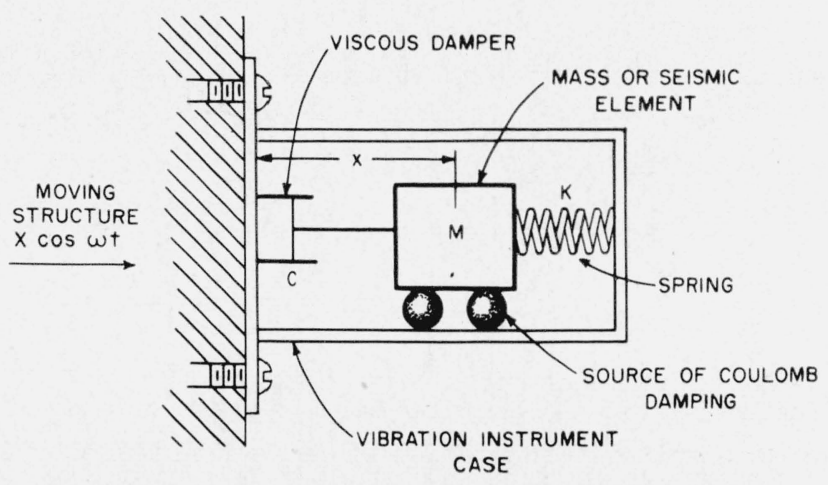

Figure 1. Typical mechanical vibration instrument. 


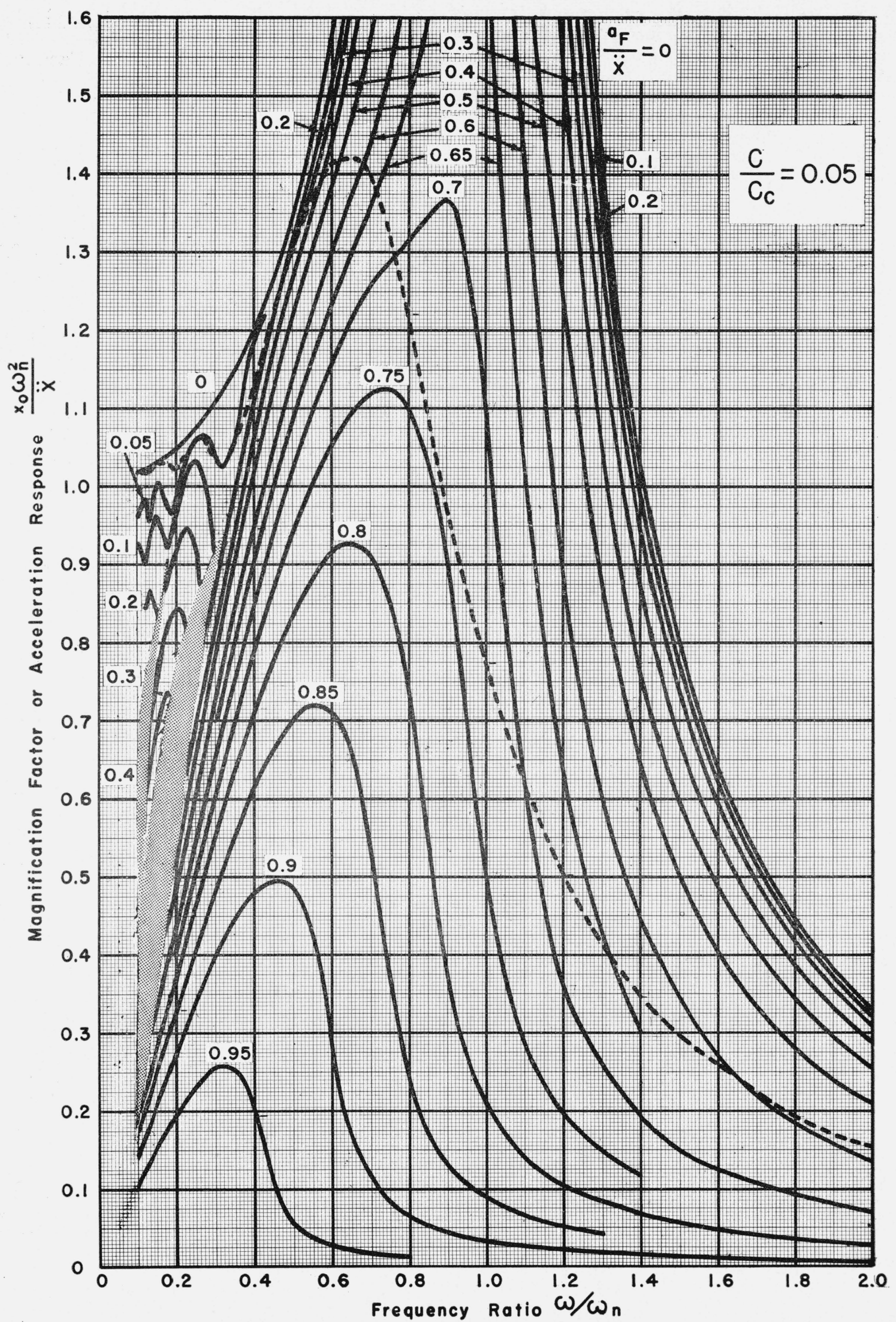

FiguRe 2. Instrument response for $C / C_{e}=0.05$. 


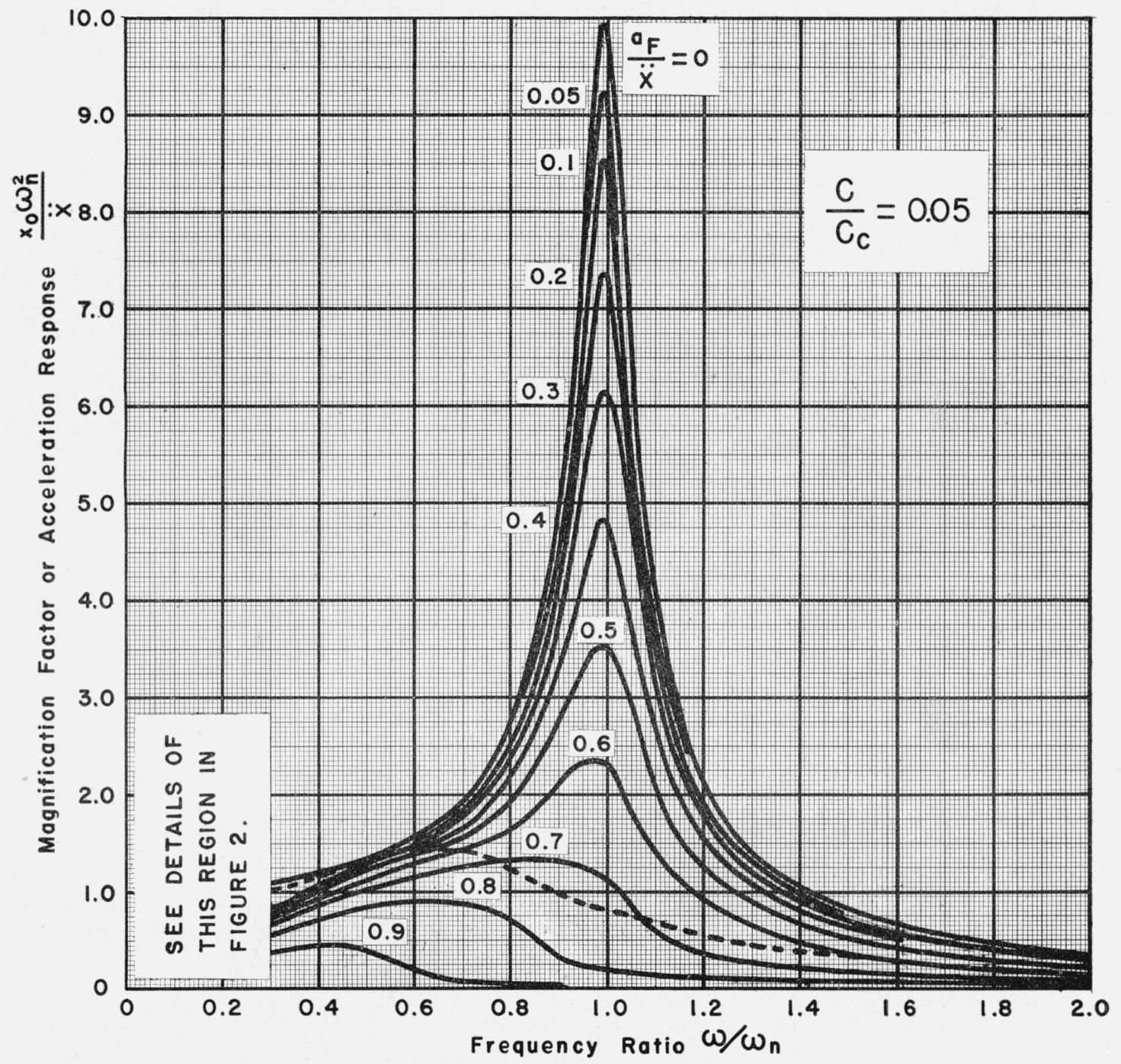

FiguRe 3. Instrument response for $C / C_{c}=0.05$. 


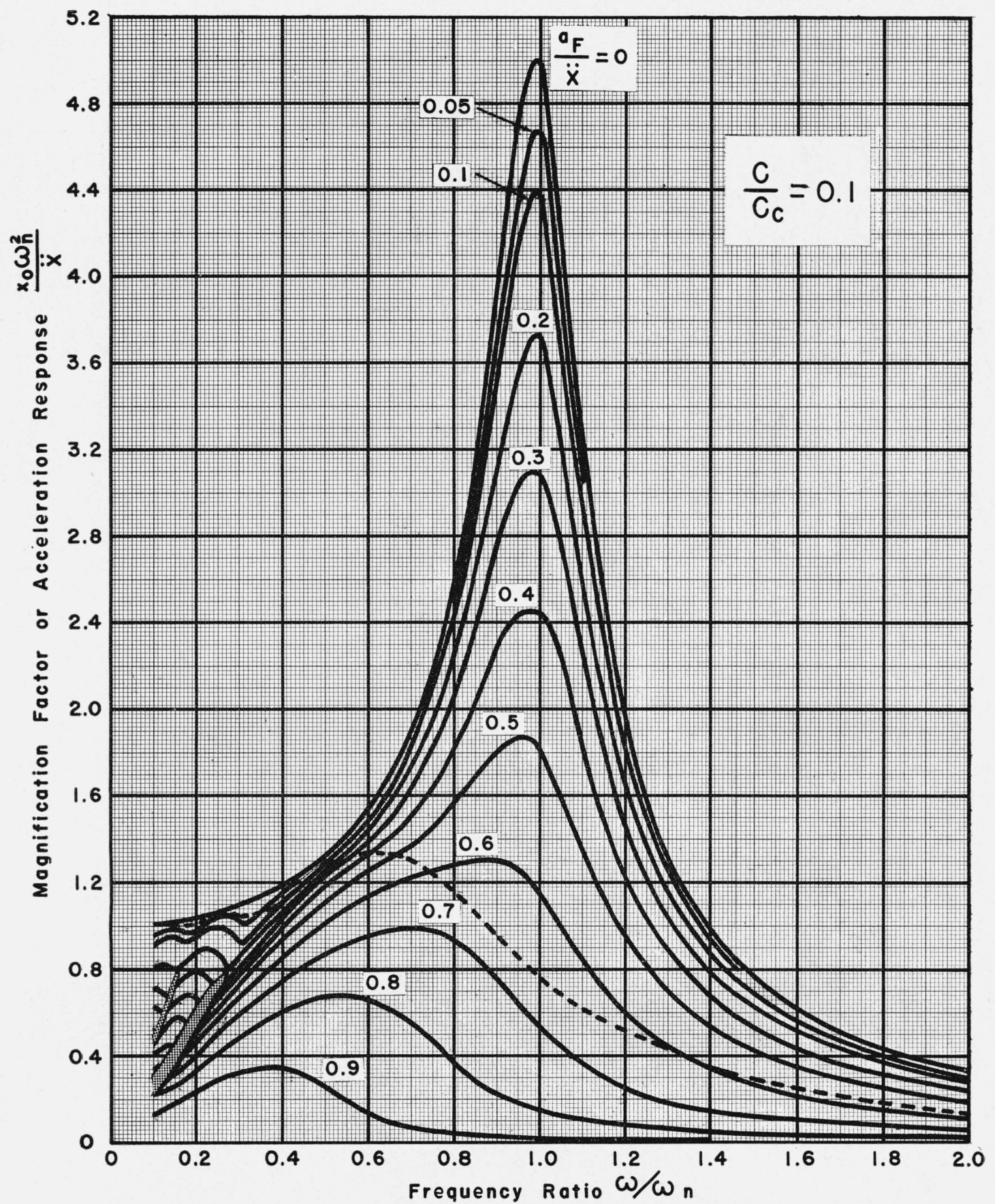

FIgure 4. Instrument response $f_{c} r \mathrm{C} / C_{c}=0.10$. 


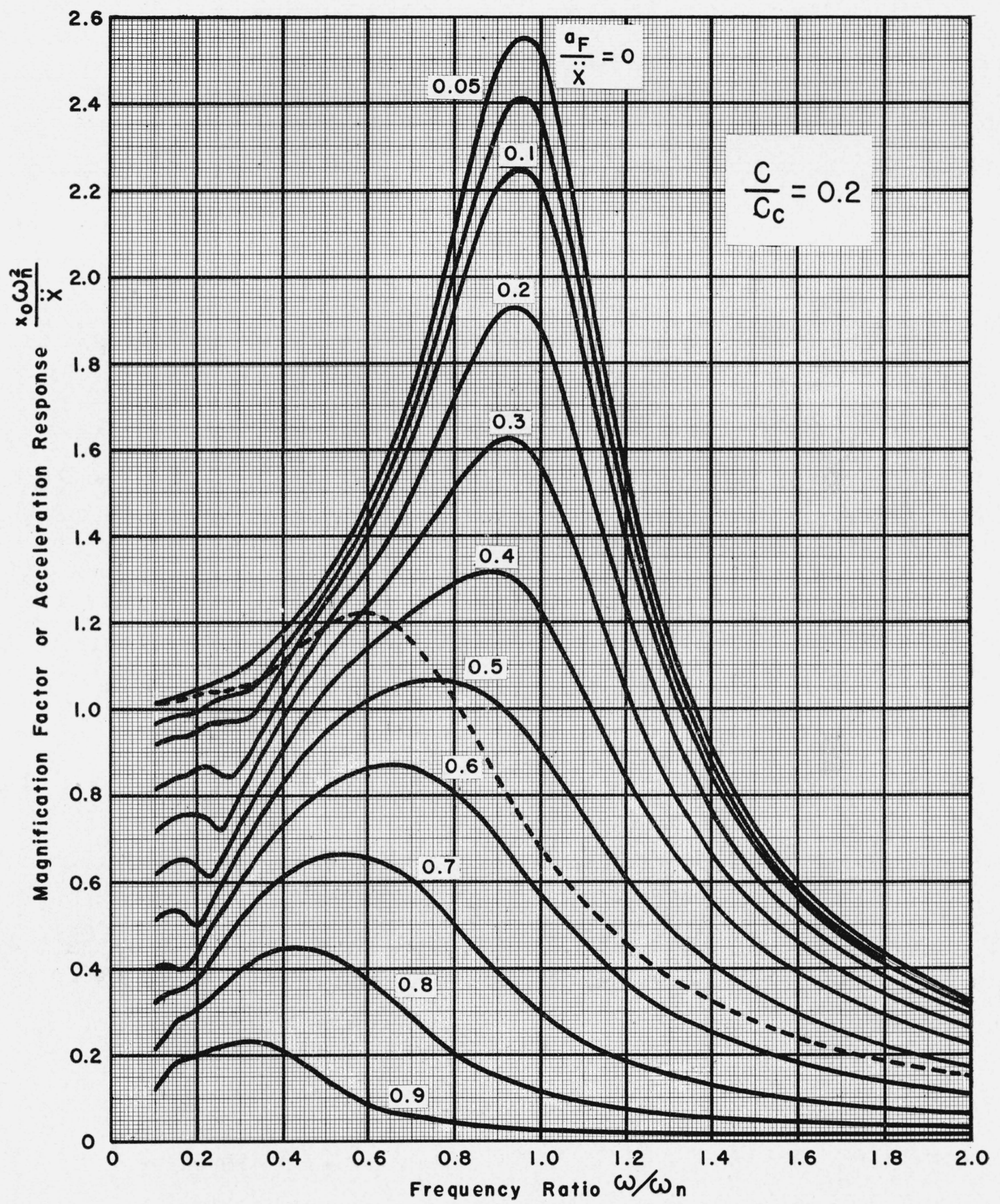

FIguRE 5. Instrument response for $C / C_{c}=0.20$. 


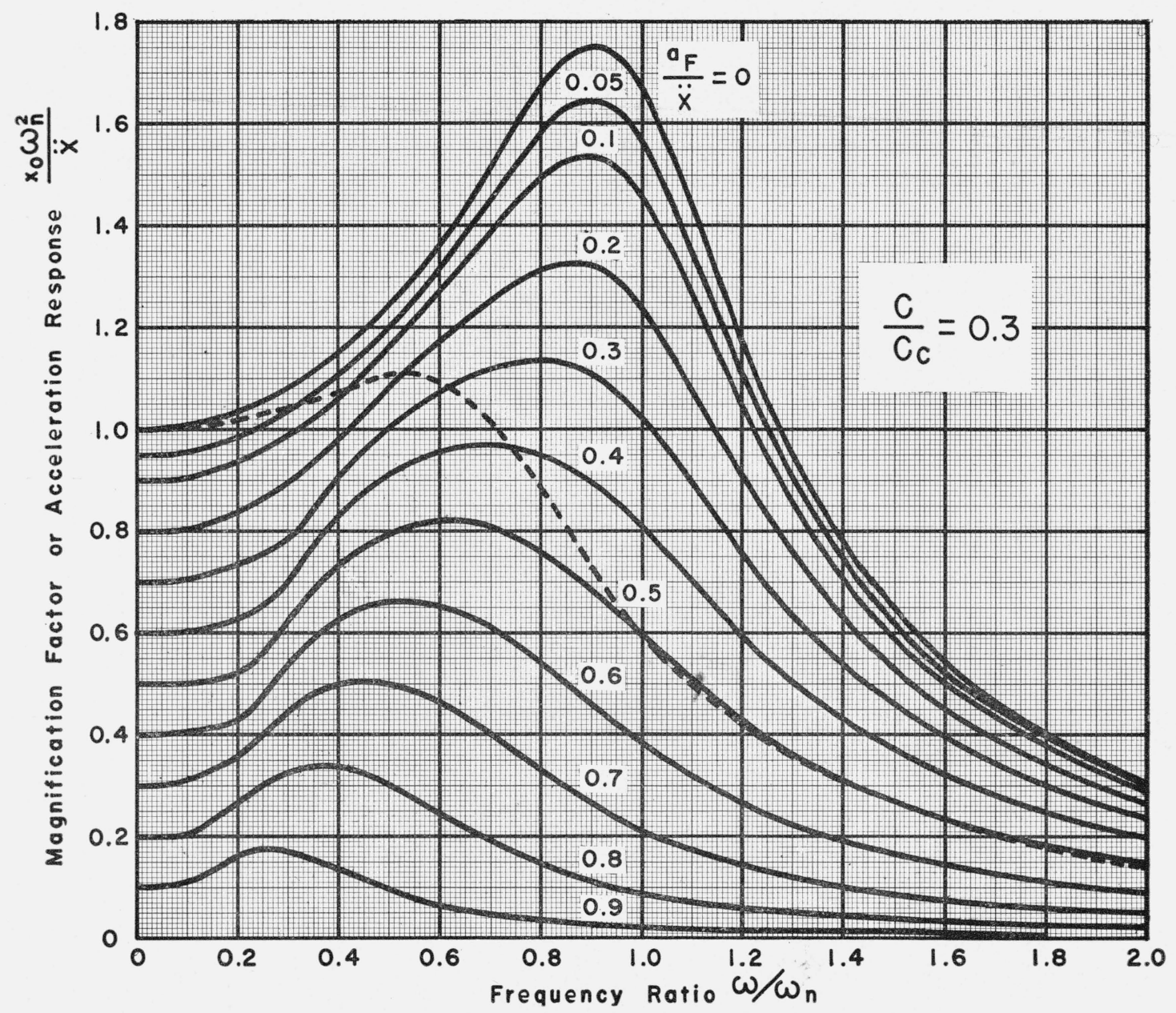

FiguRE 6. Instrument response for $C / C_{c}=0.30$. 


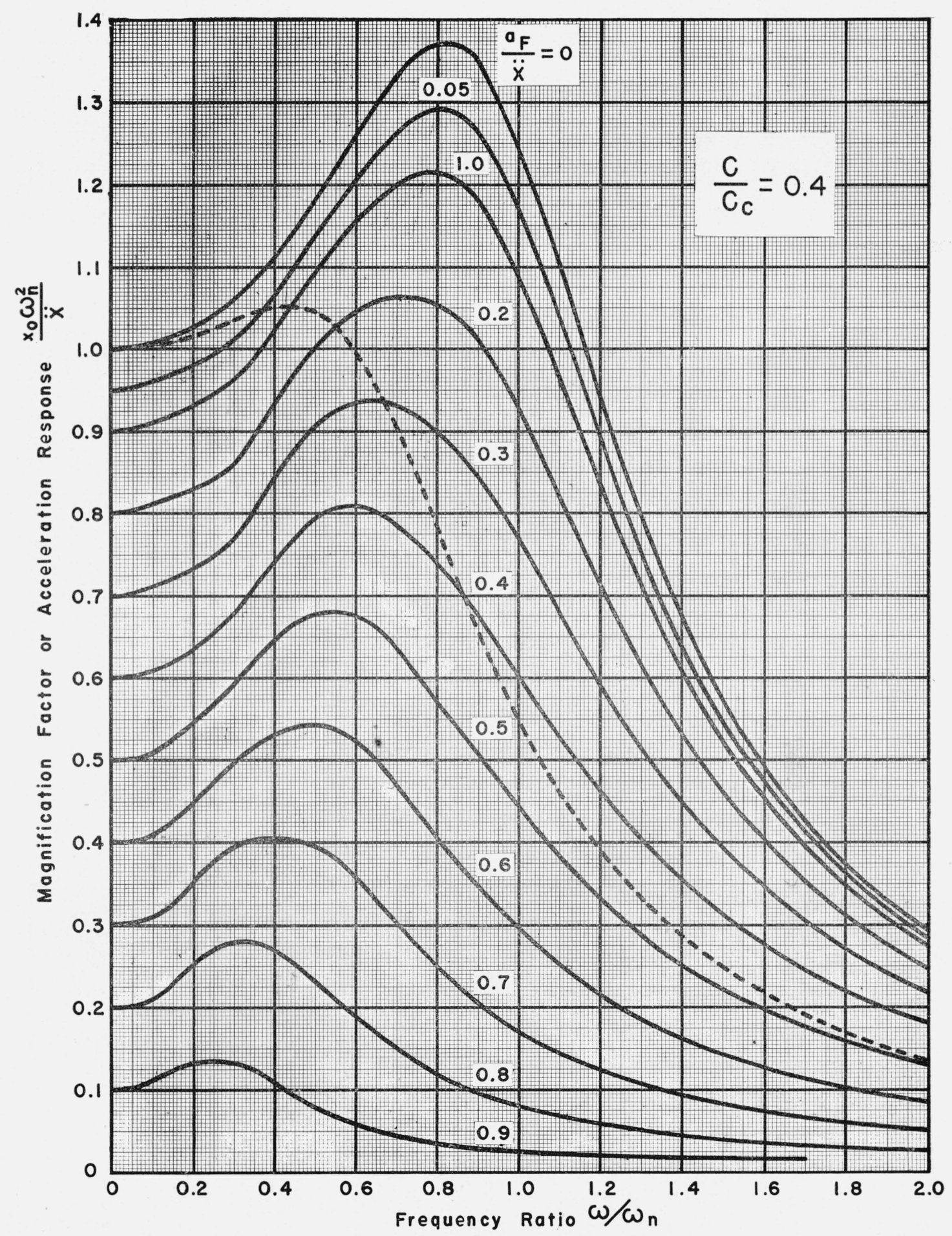

Figure 7. Instrument response for $C / C_{c}=0.40$. 


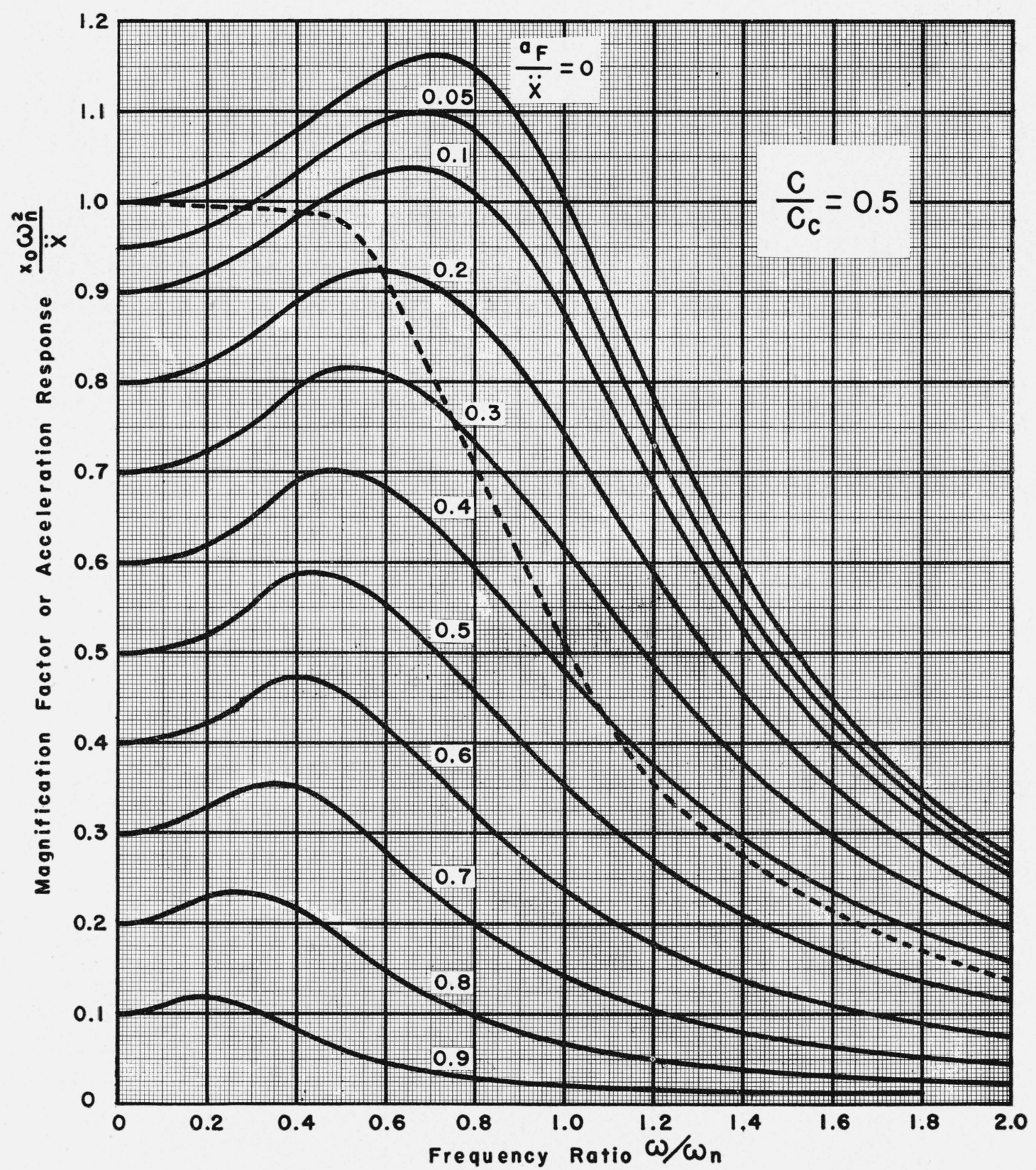

FiguRE 8. Instrument response for $C / C_{c}=0.50$. 


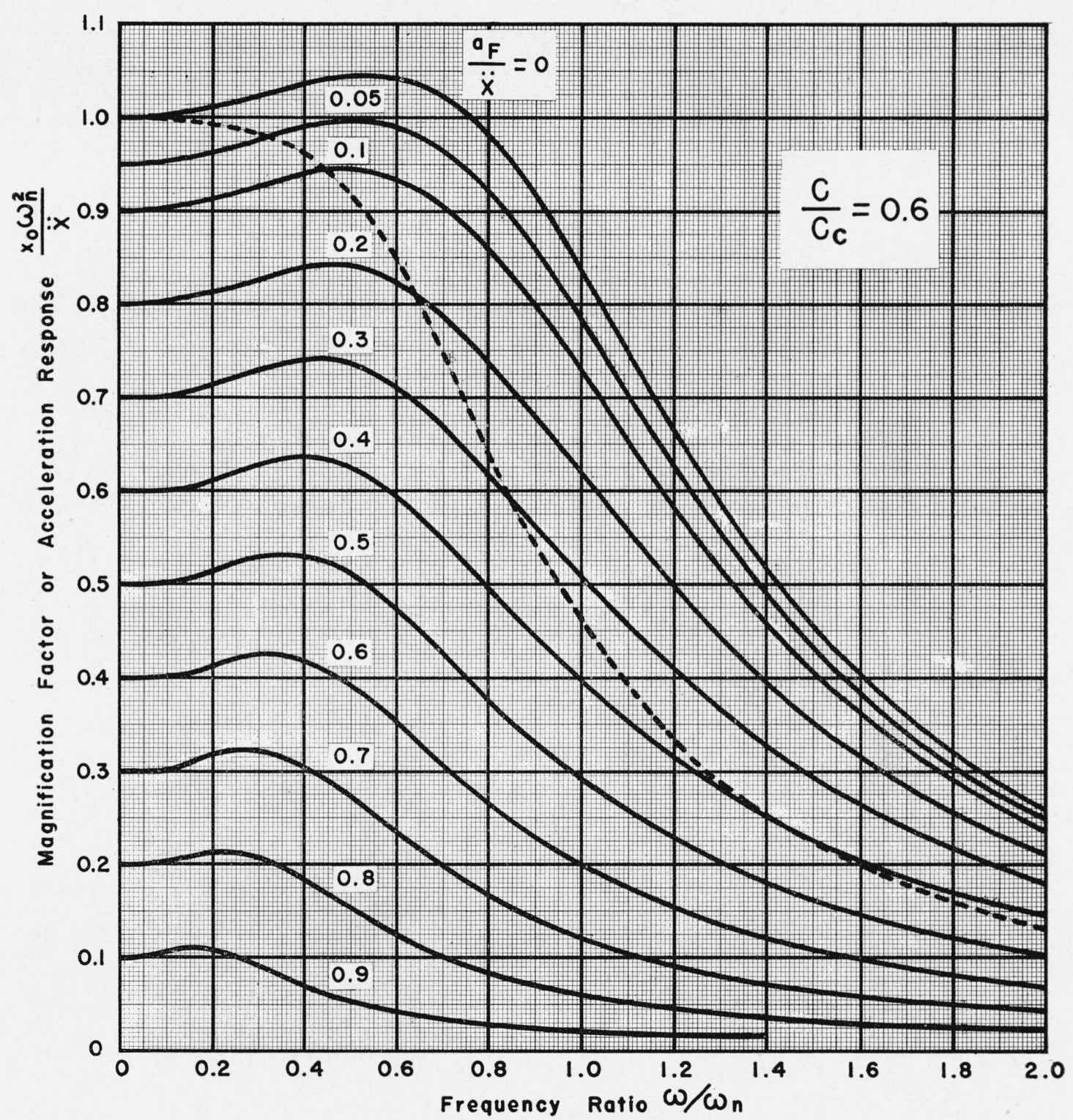

FiguRE 9. Instrument response for $C / C_{c}=0.60$. 


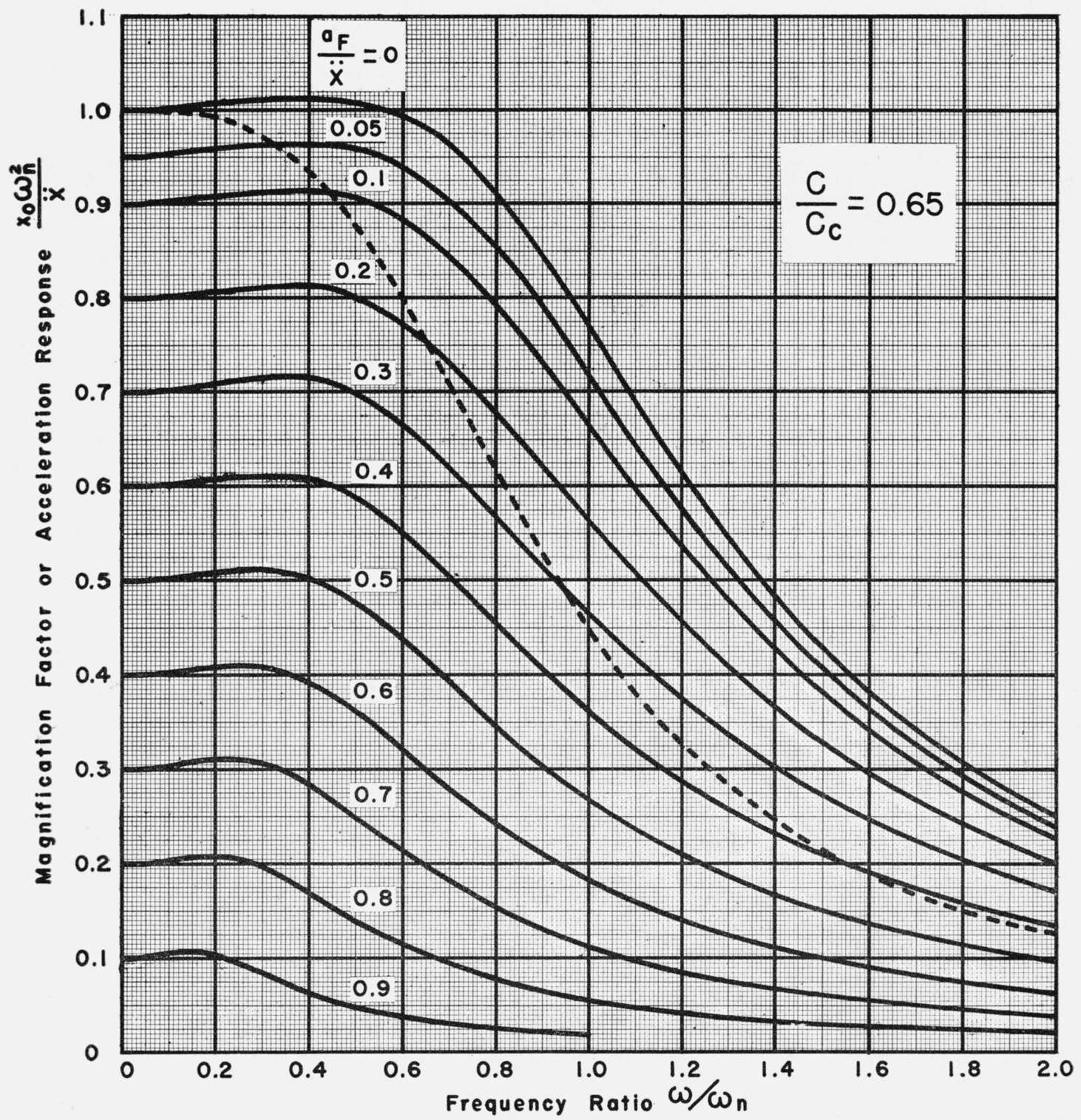

FiguRE 10. Instrument response for $C / C_{c}=0.65$. 


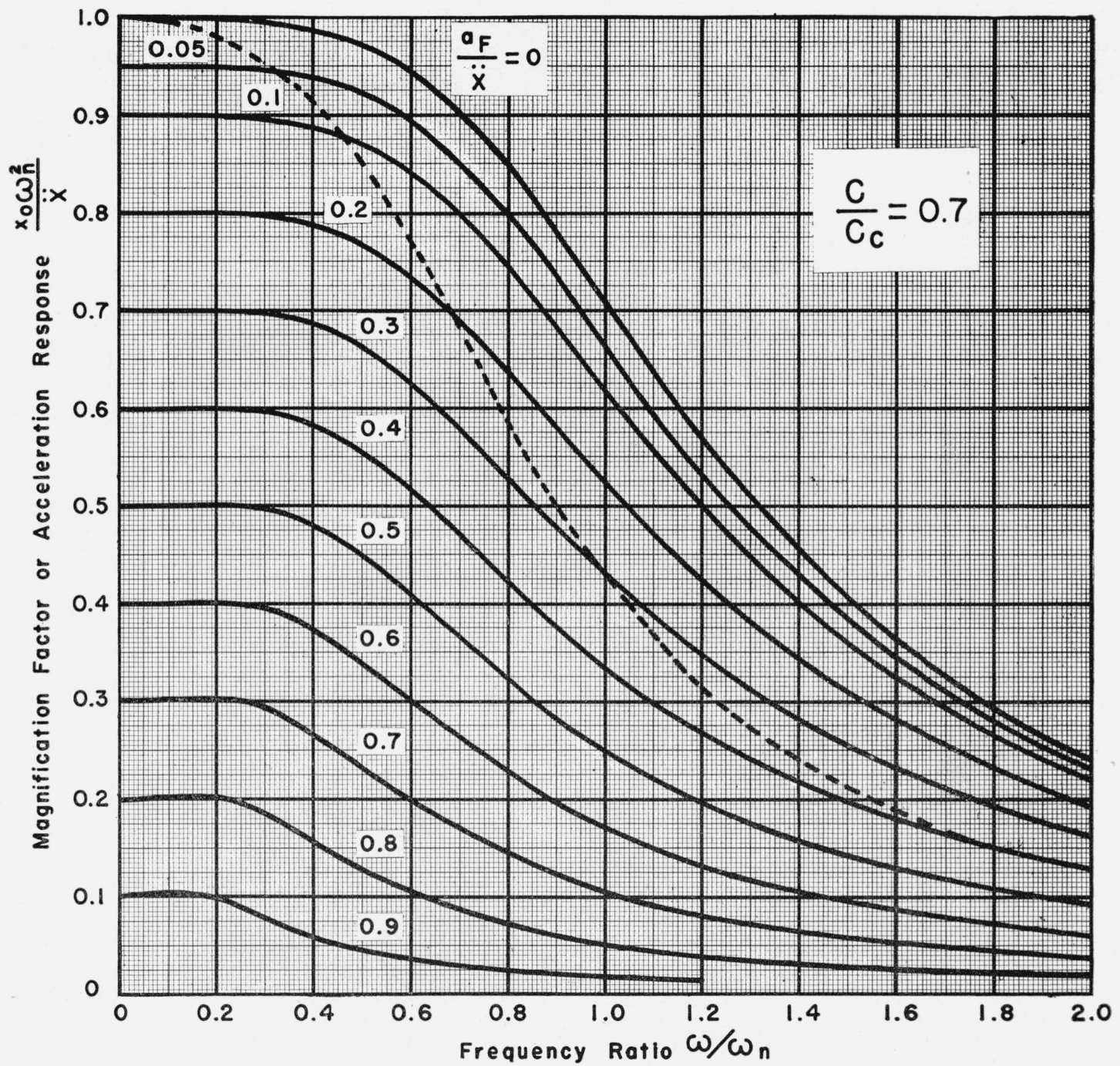

FiguRE 11. Instrument response for $C / C_{c}=0.70$. 


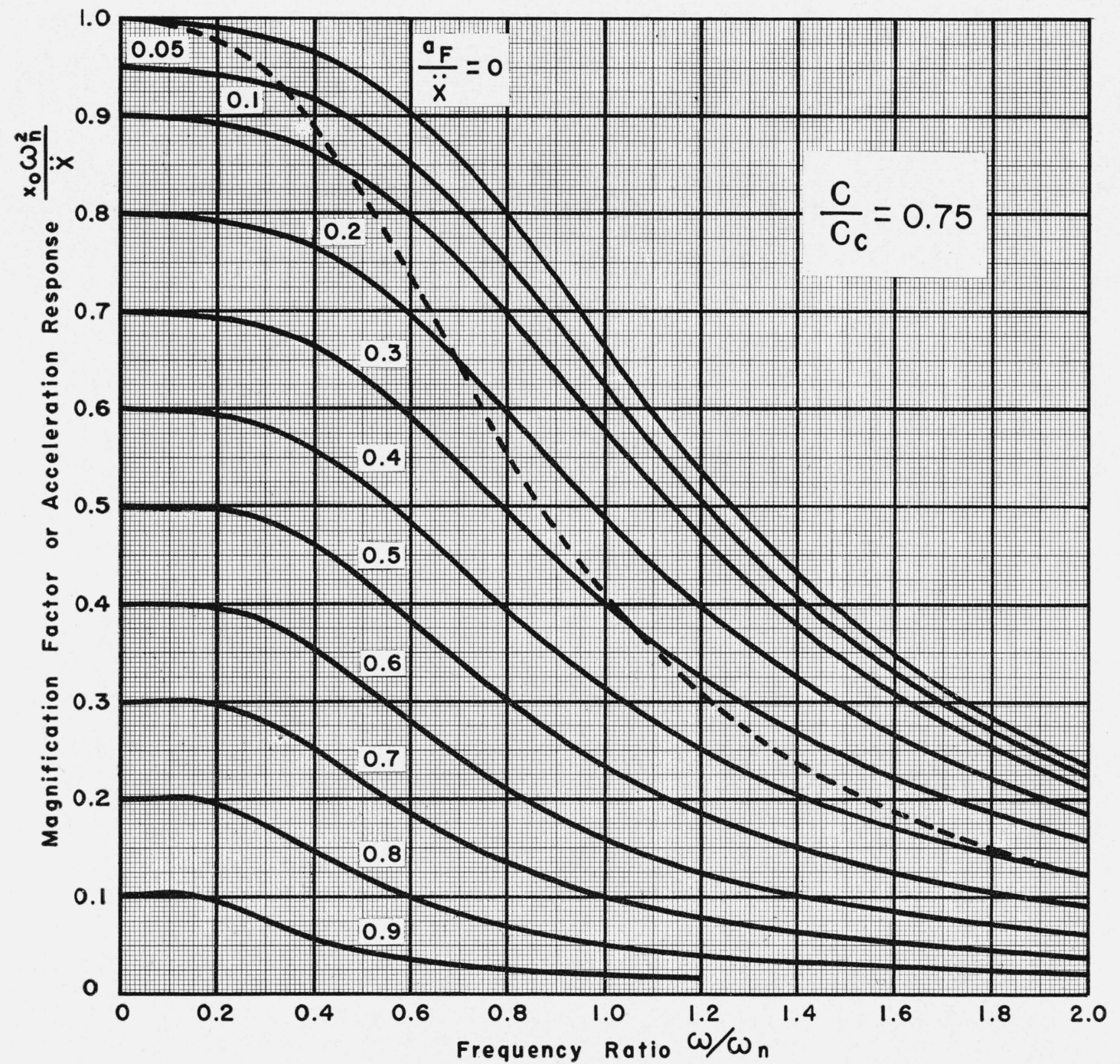

Figure 12. Instrument response for $C / C_{c}=0.75$. 


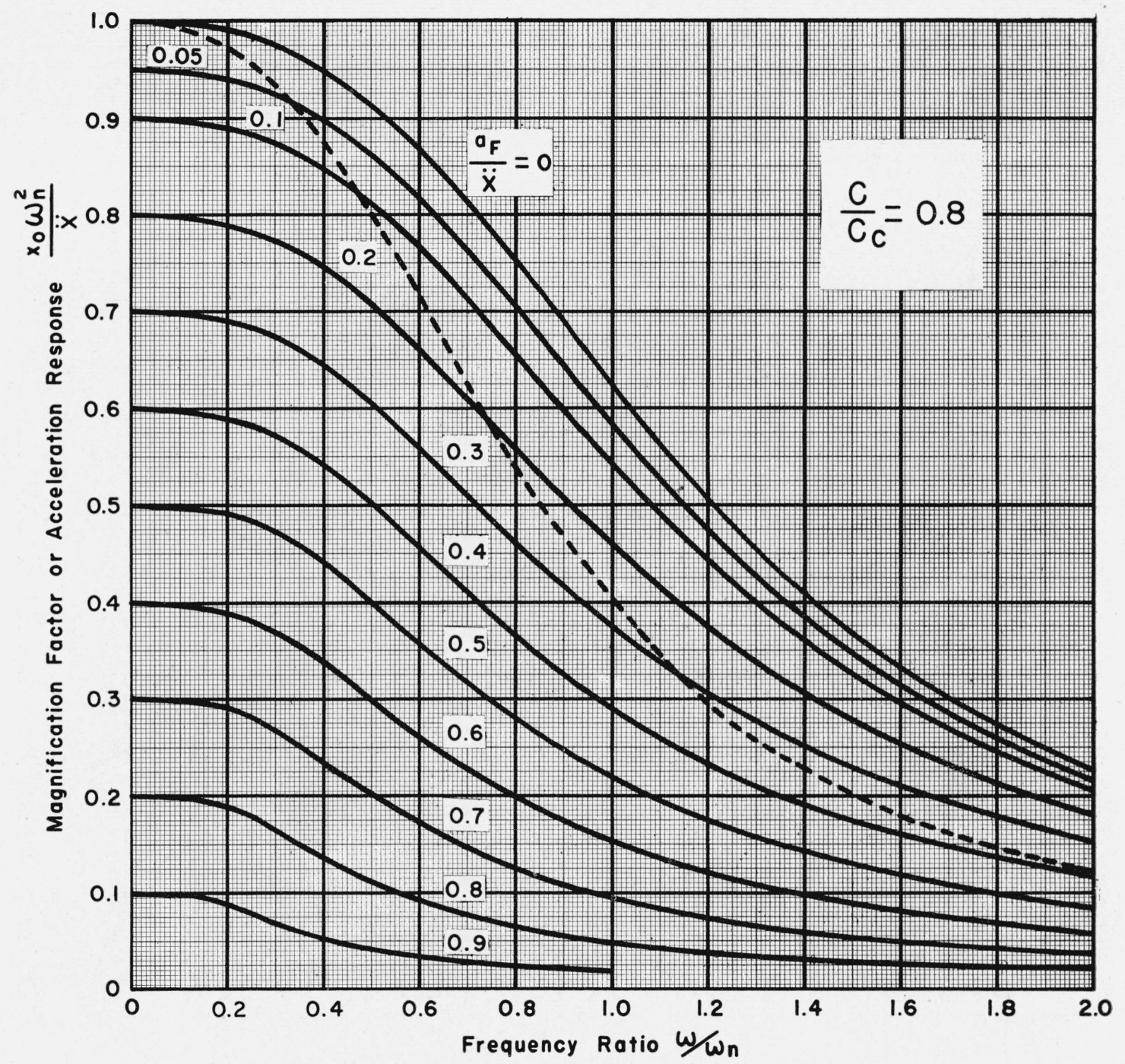

Figure 13. Instrument response for $C / C_{c}=0.80$. 


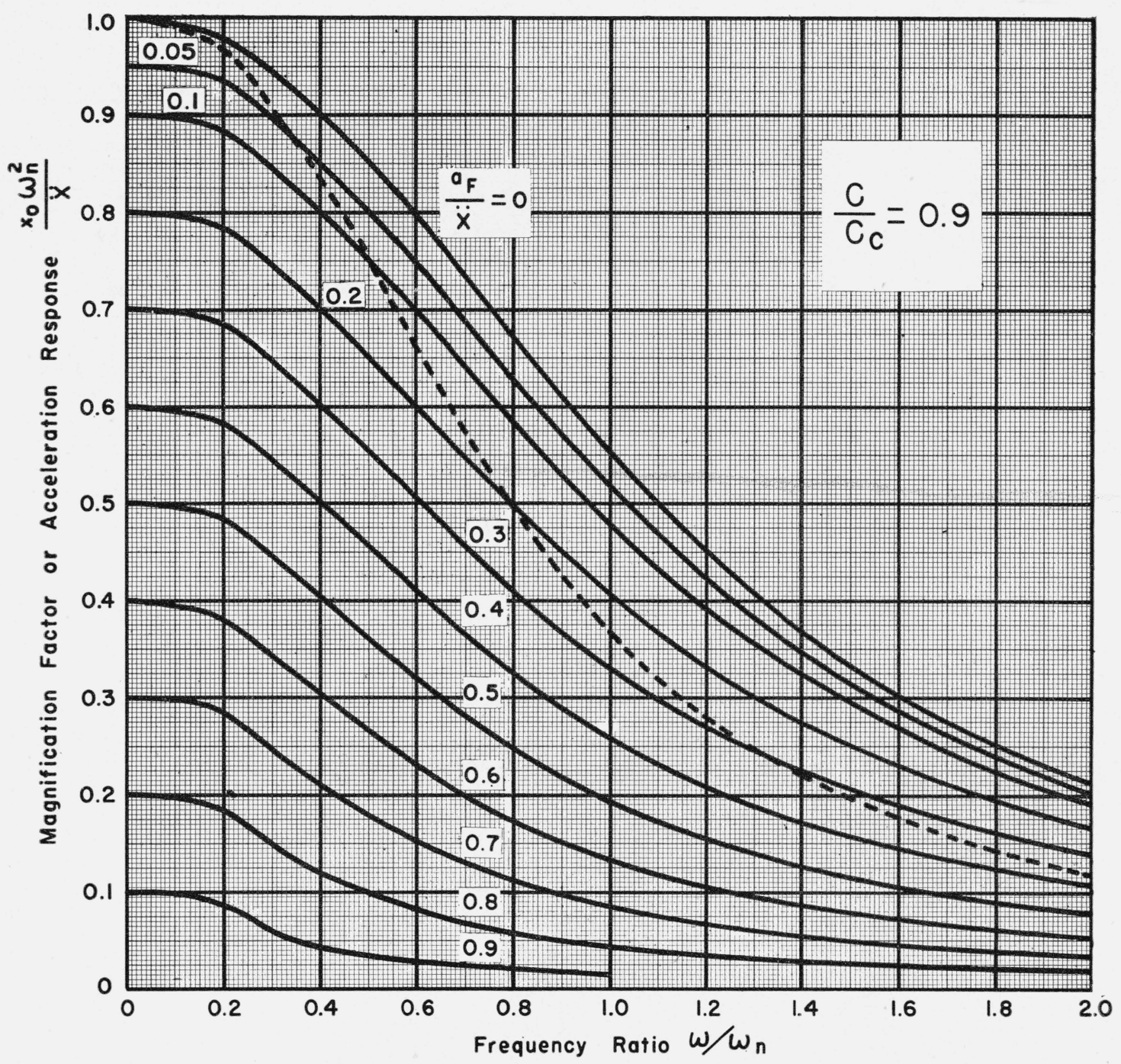

Figure 14. Instrument response for $C / C_{c}=0.90$. 


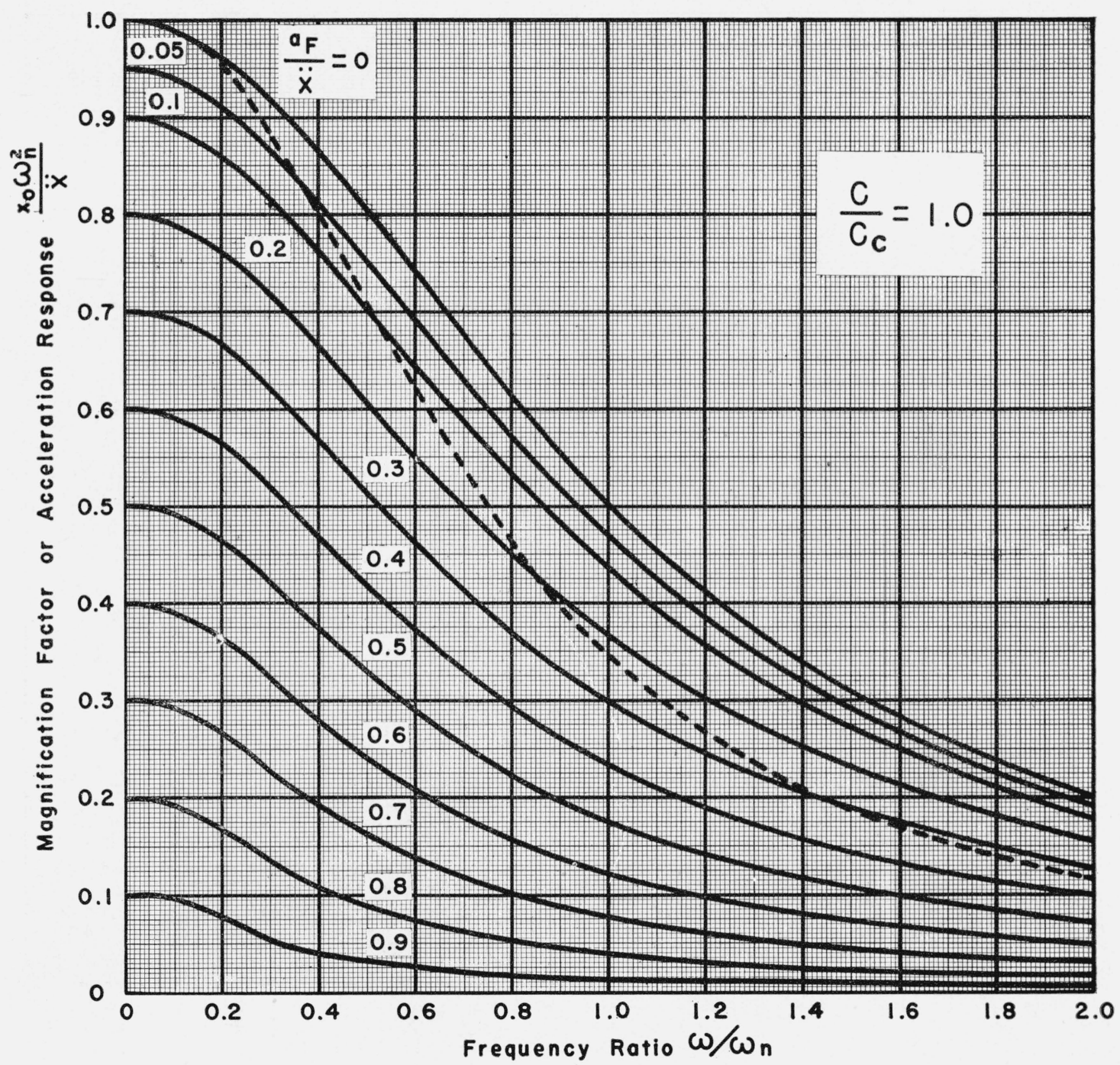

FiguRe 15. Instrument response for $C / C_{c}=1.0$. 


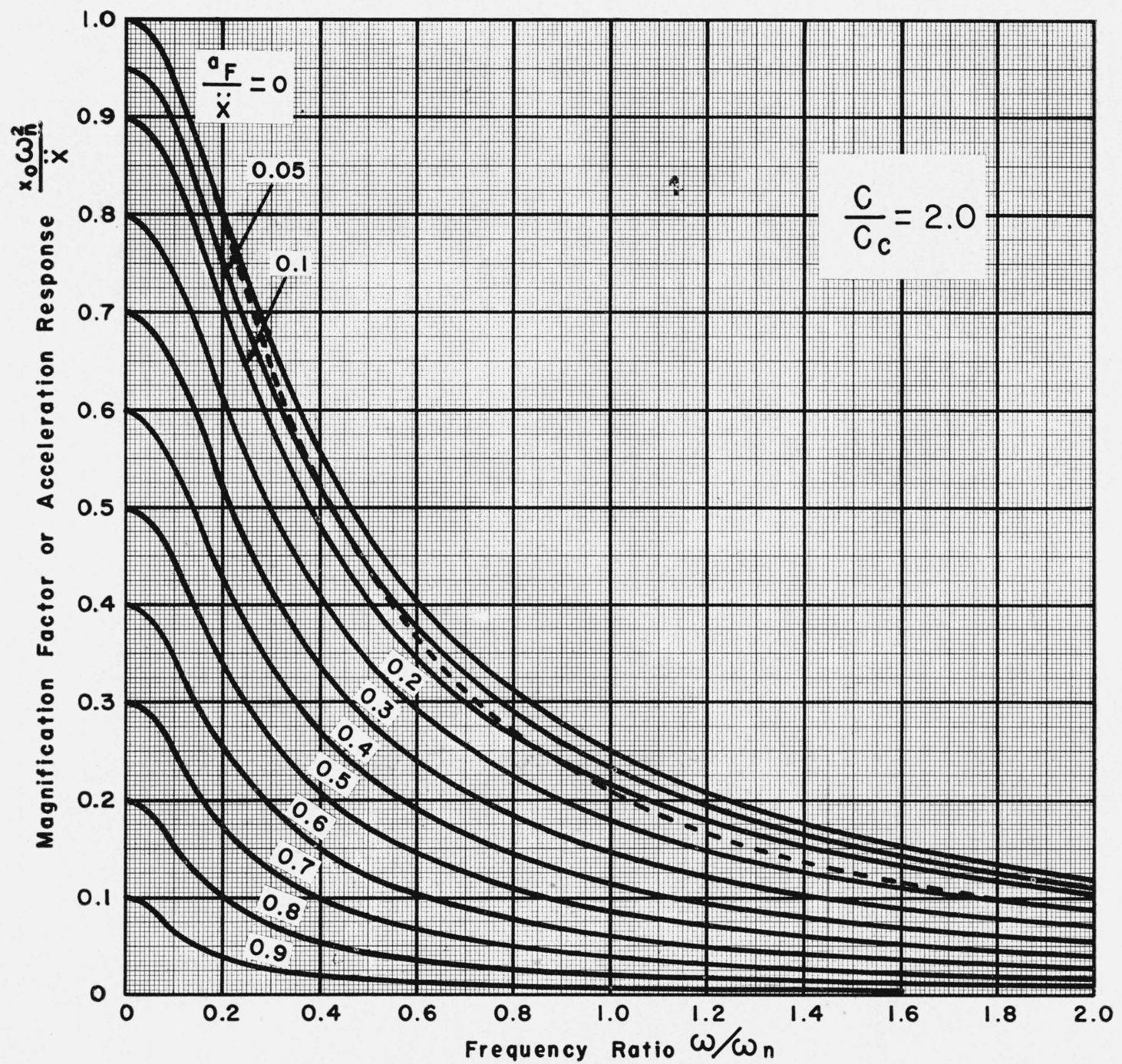

Figure 16. Instrument response for $C / C_{c}=2.0$. 


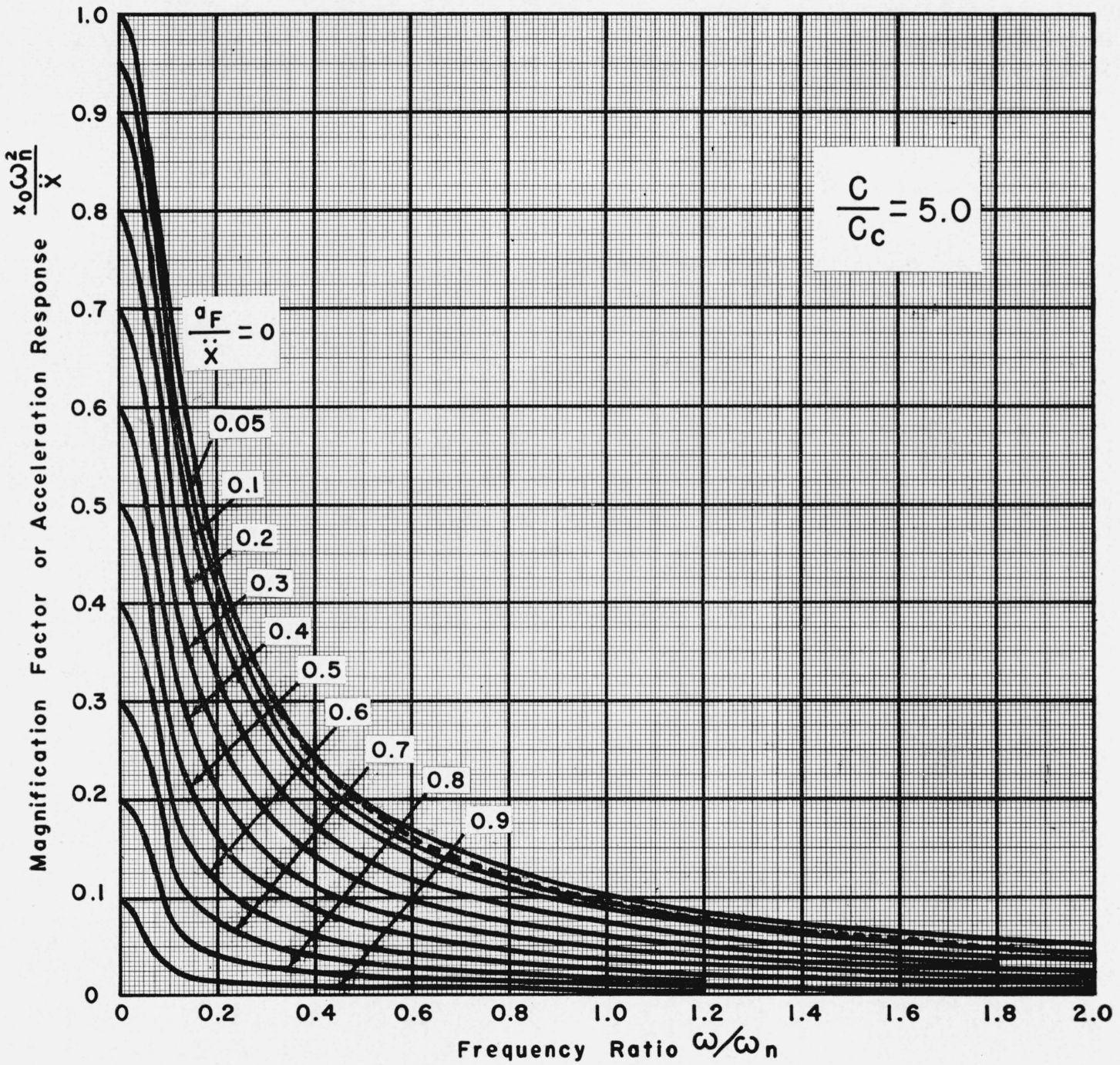

Figure 17. Instrument response for $C / C_{c}=5.0$. 

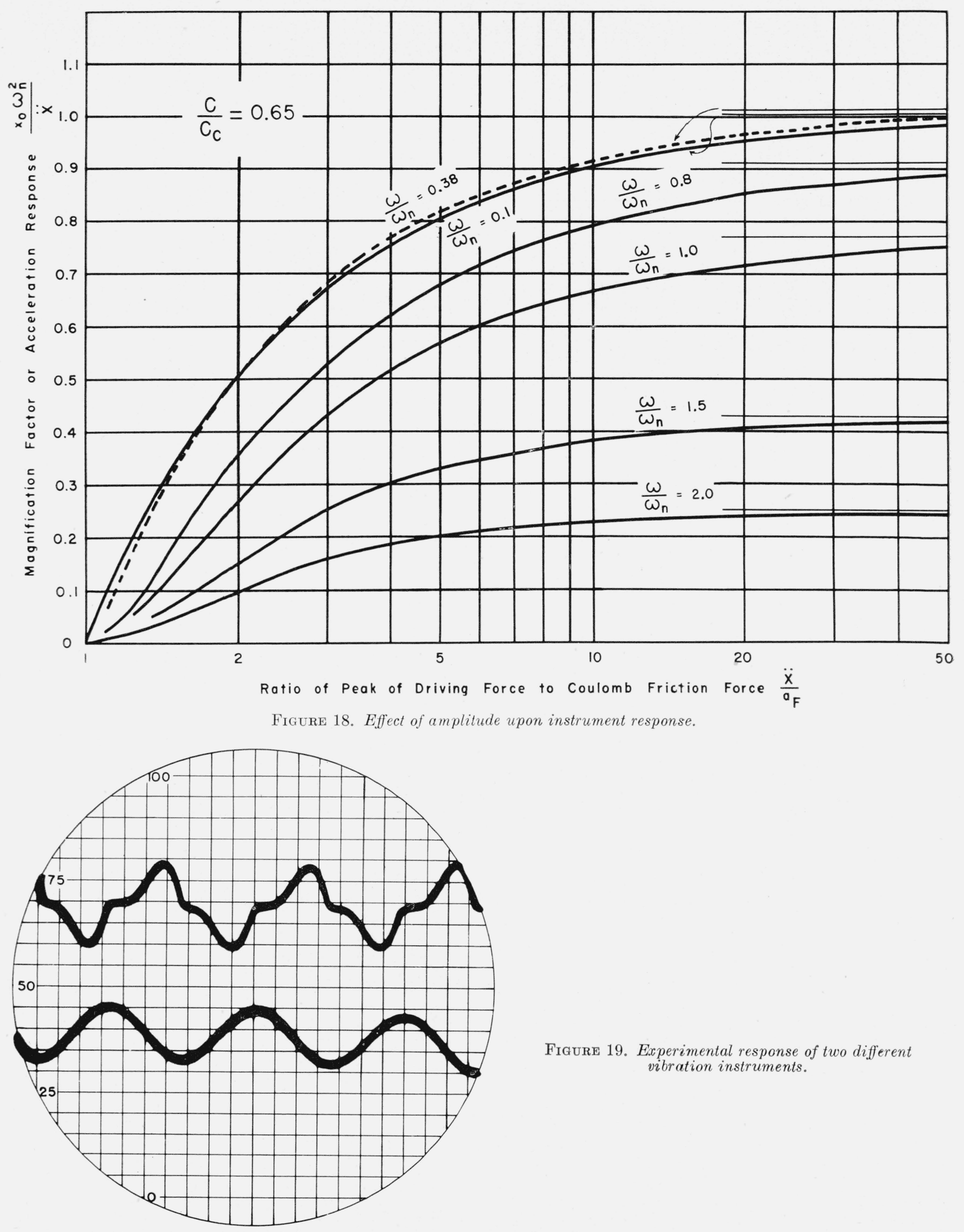

Figure 19. Experimental response of two different vibration instruments. 

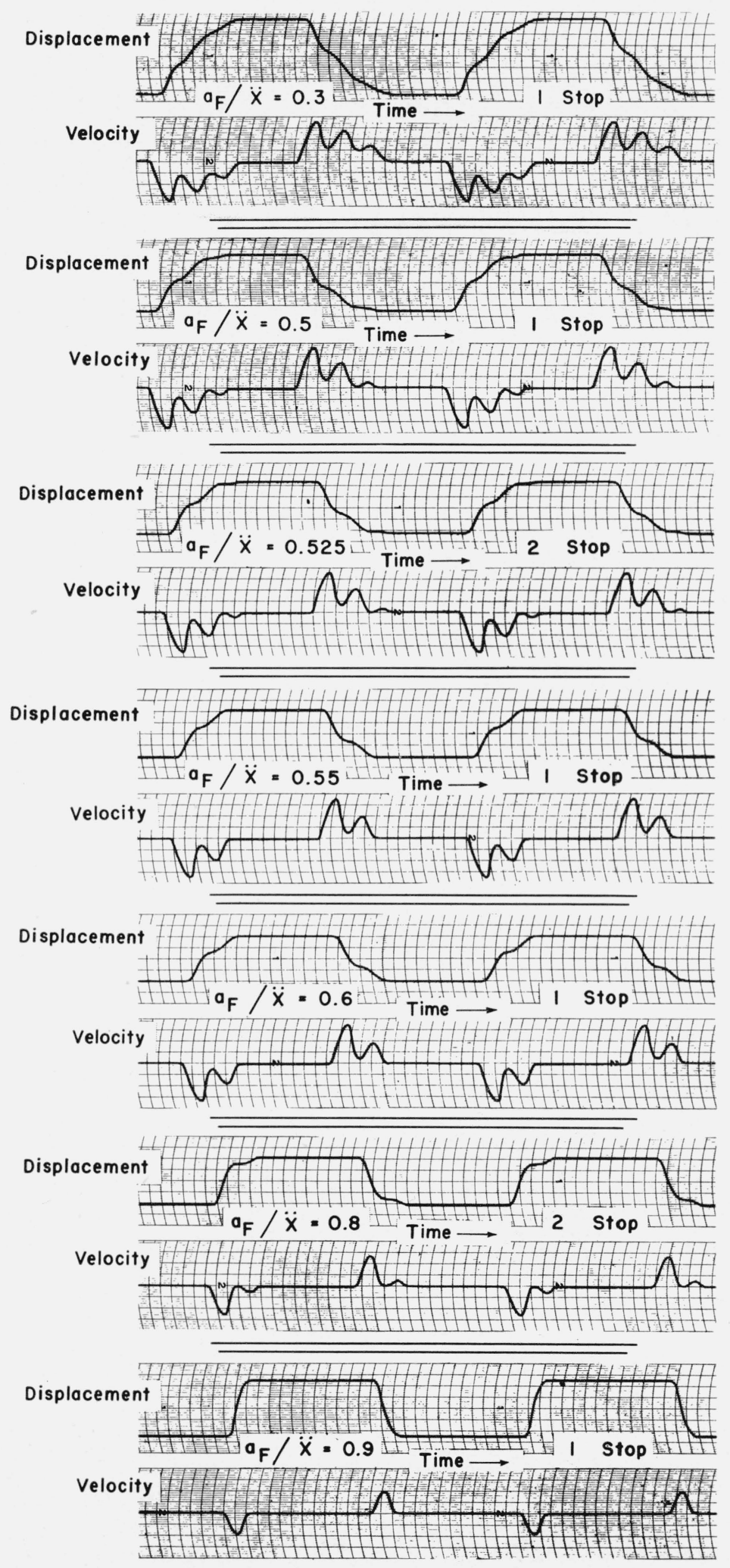

FiguRE 20. Computed response of vibration instrument.

Washington, April 3, 1956. 